\title{
Ameliorative effects of melatonin on intestinal oxidative damage in streptozotocin-induced diabetic rats
}

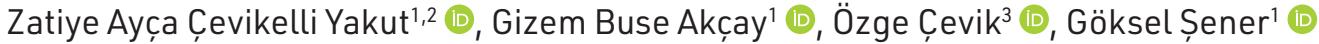 \\ 'Marmara University, Faculty of Pharmacy, Department of Pharmacology, Istanbul, Turkey \\ ${ }^{2}$ Trakya University, Faculty of Pharmacy, Department of Pharmacognosy, Edirne, Turkey \\ ${ }^{3}$ Adnan Menderes University, Faculty of Medicine, Department of Biochemistry, Aydın, Turkey
}

ORCID IDs of the authors: Z.A.C..Y. 0000-0002-6697-6781; G.B.A.0000-0002-0163-5708; Ö.Ç.0000-0002-9325-3757; G.S. 0000-0001-7444-6193

Cite this article as: Cevikelli Yakut, Z. A., Akcay, G. B., Cevik, O., \& Sener, G. (2020). Ameliorative effects of melatonin on intestinal oxidative damage in streptozotocin-induced diabetic rats. Istanbul Journal of Pharmacy. Advanced Online Publication.

\begin{abstract}
Background and Aims: The potential therapeutic effects of melatonin on changes in intestinal tissue of diabetic rats were investigated.

Methods: Male Sprague-Dawley rats were assigned into 5 groups (10 rats in each): Control, diabetes, diabetes+insulin, diabetes+melatonin, and diabetes+insulin+melatonin groups. Streptozotocin $(60 \mathrm{mg} / \mathrm{kg})$ was administered intraperitoneally to the rats to induce diabetes. At the end of 8 weeks of treatment, after blood glucose measurement and subsequent decapitation, glutathione (GSH) and malondialdehyde (MDA) levels and caspase-3, myeloperoxidase (MPO), and superoxide dismutase (SOD) activities in the intestinal tissue were investigated.

Results: In diabetic animals, elevated blood glucose levels caused oxidant damage in the intestinal tissue that was demonstrated with increased MDA levels, caspase and MPO activities, and decreased GSH levels and SOD activities. Although melatonin demonstrated more significant results than insulin, separate administration of both melatonin and insulin improved the oxidative damage parameters compared to the diabetes group. In the combined treatment group, all parameters were back to control levels statistically more significant when compared with the treatment-alone.

Conclusion: Melatonin has been shown to protect intestinal tissue from diabetic oxidant damage. With insulin treatment in type I diabetes, melatonin supplements may increase the quality of life through reducing complications.
\end{abstract}

Keywords: Diabetes mellitus, oxidative damage, intestine, insulin, melatonin

\section{INTRODUCTION}

Diabetes Mellitus (DM) is characterized by high blood glucose levels which are caused by the impaired effect and/or release of insulin. Since hyperglycemia triggers the oxidative stress that plays a role in many diabetes complications (Garcia, Rodrigues, Alonso, Rodrigues-Ochoa, \& Aguilar, 2015), whether antioxidants have positive effects on DM is an object of interest.

Free radicals that are necessary for many signals in physiological processes, such as differentiation and migration in cells (Bansal \& Bilaspuri, 2011), trigger the cell damage through the passage of unpaired electrons into proteins and DNAs (Vural, Sabuncu, Arslan, \& Aksoy, 2001). While antioxidants found endogenously or taken exogenously provide defense against this damage, several factors, such as aging, various drugs, and diseases, make the body prone to oxidative stress by disrupting the balance between

Address for Correspondence:

Göksel ȘENER, e-mail: gsener@marmara.edu.tr

This work is licensed under a Creative Commons Attribution 4.0 International License. 
antioxidants and pro-oxidants (Ullah, Khan, \& Khan, 2016). Hyperglycemia triggers oxidative stress by increasing free radical formation and suppressing the antioxidant defense system (Ighodaro, 2018). The oxidative stress in diabetes damages almost all organs in the body, such as the heart, kidneys, eyes, neurons, liver, small and large vessels, and gastrointestinal (GI) system (Kochar \& Umathe, 2009). Diabetes mellitus can affect all regions of Gl tract, such as the esophagus, intestines, and anorectum (Wolosin \& Edelman, 2000), due to the elevated free radical levels. Diabetes mellitus generates morphological and functional changes in the intestinal mucosa; for instance, hyperplasia and hypertrophy occur in epithelial cells (Bhor, Raghuram, \& Sivakami, 2004).

Antioxidants are molecules that can prevent tissue damage by reducing the amount and effectiveness of reactive oxygen species (ROS) (Vural et al.,2001). In recent reports, free radical scavenging and antioxidant properties of melatonin, which is a pineal hormone regulating circadian rhytm, have been indicated, in addition to its positive effects on glucose metabolism (Alikhani, Keshavarzi, Hadjzadeh, \& Karimi, 2015; Hadjzadeh, Alikhani, Hosseinian, Zarei, \& Keshavarzi, 2018; Yang et al., 2016). Melatonin protects against oxidant damage because melatonin stimulates the antioxidant enzyme production, neutralizes the reactive oxygen and nitrogen species, reduces the proinflammatory cytokine levels, and stabilizes the cell membrane (Esposito \& Cuzzocrea, 2010). Moreover, curative effects of melatonin on gastric and intestinal problems in different experimental studies of DM throw light on our study (Alikhani et al., 2015; Hadjzadeh et al., 2018; Yang et al.,2016).

The present study aims to determine the possible benefits of melatonin supplementation to protect the intestinal tissues from oxidative injury in streptozotocin (STZ)-induced diabetic rats.

\section{MATERIALS AND METHODS}

\section{Animals and ethics}

Three months old male Spraque-Dawley rats (250-300 grams) were obtained from Marmara University Research Center for Experimental Animals. All experimental protocols were performed according to the Marmara University Animal Care and Use Committee (Protocol: 33.2015.mar).

\section{Experimental protocol}

The rats were randomly assigned into five groups (10 rats in each): Control group (C), diabetes group (D), insulin-treated diabetes group $(D+I)$, melatonin-treated diabetes group $(D+M)$, and insulin-and-melatonin-treated diabetes group (D+I+M). In the diabetes groups, STZ $(60 \mathrm{mg} / \mathrm{kg})$ was applied intraperitoneally (ip) to induce diabetes. The rats demonstrating blood glucose levels above $200 \mathrm{mg} / \mathrm{dL}$ were regarded as diabetic (Husni, Anggaara, Isnansetyo, \& Nugroho, 2016). Following the diabetes induction, rats were treated with insulin (sc, 6U/ kg) (Paskaloğlu, Şener, \& Ayanoğlu-Dülger, 2004) and/or melatonin (ip, 10 mg/kg) (Alikhani et al., 2015; Hadjzadeh et al., 2018; Yang et al., 2016). Treatments were continued for 8 weeks (Paskaloğlu et al., 2004). $3 \mathrm{ml}$ of $0.9 \% \mathrm{NaCl}$ solution was also applied daily to all groups for fluid replacement. Body weights and blood glucose levels were recorded after the diabetes in- duction and at the end of the $8^{\text {th }}$ week. Blood glucose levels were measured with a glucometer (Accu-Chek, F. Hoffman-La Roche Ltd, Basel, Switzerland) by taking blood samples from the orbital vein under mild ether anesthesia. Eight weeks later, intestinal tissue specimens of the animals were removed to detect malondialdehyde (MDA) and glutathione (GSH) levels, myeloperoxidase (MPO), superoxide dismutase (SOD), and caspase-3 activities. In addition, western blotting was performed to evaluate caspase-3 protein expression.

\section{Measurements in intestinal tissue samples Malondialdehyde (MDA) and glutathione (GSH) levels}

To evaluate the MDA and GSH levels, intestinal tissue specimens were homogenized in an ice-cold medium containing $150 \mathrm{mM} \mathrm{KCl}$. Beuge and Aust's method was used for MDA assay (Beuge \& Aust,1978). A solution containing thiobarbituric acid (\% 0.375, TBA) and trichloroacetic acid (15\%, TCA) in $0.25 \mathrm{~N}$ $\mathrm{HCl}$ was added to $0.5 \mathrm{~mL}$ homogenate. The absorbance of the color of the samples cooled and centrifuged after being kept in a hot water bath for 15 minutes was read at $532 \mathrm{~nm}$. The unit used was $\mathrm{nmol} / \mathrm{g}$ tissue.

The Beutler method was used to investigate the GSH levels (Beutler, Duron, \& Kelly, 1963). $0.4 \mathrm{~mL}$ 10\% homogenate was mixed with $0.2 \mathrm{~mL} 20 \%$ TCA and centrifugated for 15 minutes at $3000 \mathrm{rpm}$. Supernatant $(0.5 \mathrm{~mL})$ was collected and added to a $0.3 \mathrm{~mol} / \mathrm{L} \mathrm{Na} \mathrm{NPO}_{4} \cdot 2 \mathrm{H}_{2} \mathrm{O}$ solution (2 mL). Afterwards a 0.2 $\mathrm{mL}$ dithiobisnitrobenzoate solution $(0.4 \mathrm{mg} / \mathrm{mL})$ in $1 \%$ sodium citrate was added. The absorbance was recorded at $412 \mathrm{~nm}$ after mixing. Glutathione levels were calculated by the aid of the extinction coefficient of $1.36 \times 10^{4} \mathrm{M}^{-1} \mathrm{~cm}^{-1}$. The unit used was $\mu \mathrm{mol} / \mathrm{g}$ tissue.

\section{Superoxide dismutase (SOD) activity}

According to the method of Mylroie et al., the absorbance of color formed in samples kept in the fluorescence light and at $37{ }^{\circ} \mathrm{C}$ with the buffer prepared by using $50 \mathrm{mM}$ potassium phosphate buffer-EDTA, $0.2 \mathrm{mM}$ ribofilavin, and $6 \mathrm{mM}$ o-dianisinide was read at $460 \mathrm{~nm}$. The unit used was U/g tissue (Mylroie, Collins, Umbles, \& Kyle, 1986).

\section{Myeloperoxidase (MPO) activity}

Tissue specimens were homogenized in a $50 \mathrm{mM}$ potassium phosphate buffer (PB) (pH 6.0). For 10 min, centrifugation was carried out at 41,400 g. Afterwards the pellets were suspended in a $50 \mathrm{mM}$ PB solution which contained $0.5 \%$ hexadecyltrimethylammonium bromide. Centrifugation was carried out at $41,400 \mathrm{~g}$ for $10 \mathrm{~min}$ after three freeze-thaw cycles. Sonication was performed between the cycles. $0.3 \mathrm{~mL}$ of aliquots were added to a $2.3 \mathrm{~mL}$ reaction mixture (o-dianisidine, $50 \mathrm{mM} \mathrm{PB}$, and $20 \mathrm{mM} \mathrm{H}_{2} \mathrm{O}_{2}$ solution). The absorbance change at $460 \mathrm{~nm}$ for 3 min on a spectrophotometer was used to calculate the MPO activity (DU-73 Spectrophtometer, Beckman Coulter Inc, Fullerton, CA, USA). The unit used was U/g tissue (Hillegas, Griswold, Brickson, \& Winslow, 1990).

\section{Caspase-3 activity}

Caspase-3 activities in the tissue samples were investigated in accordance with the kit procedure, using Colorimetric Protease Assay Kit of Caspase-3 (Caspase-3 Colorimetric Kit-200 
Tests Biosource (TM), Thermo Fisher Scientific Inc, Waltham, MA, USA). Formation of chromophore p-nitroanilide from caspase-3 substrate N-Acetyl-Asp-Glu-Val-Asp p-nitroanilide was evaluated spectrophotometrically. The absorbance of released p-nitroanilide was determined in an ELISA reader at $405 \mathrm{~nm}$ (Çevik et al., 2012).

A cell blasting buffer was added to the tissue homogenate and suspended in a cold environment, and the supernatant was used after centrifugation at 10,000 g. The supernatant was loaded onto the wells. After adding $50 \mu \mathrm{L}$ of reaction buffer, 4 mM N-Acetyl-Asp-Glu-Val-Asp p-nitroanilide substrate was added. After the incubation for 2 hours at $37^{\circ} \mathrm{C}$, the results were calculated according to the standards by reading in the ELISA reader at $405 \mathrm{~nm}$.

\section{Caspase-3 protein expression analysis with western blot method}

Samples were blasted with $70 \%$ amplitude in ice ultrasonic homogenizer for 15 seconds by using a cell blasting buffer. In the upper phase obtained with centrifugation at $12,000 \mathrm{~g}$ at $40^{\circ} \mathrm{C}$, western blotting studies were carried out (Bradford, 1976).

The protein $(25 \mu \mathrm{g})$ was resolved on the $12 \%$ SDS-PAGE. Next, it was transferred to a nitrocellulose membrane (Santa Cruz Biotechnology Inc, CA, USA). In order to block the nitrocellulose membrane, 5\% nonfat skim milk powder in tris-buffered saline (TBS) was used (Sigma-Aldrich, St. Louis, Missouri, USA). Then the nitrocellulose membrane was washed twice with $0.1 \%$ Tween-20 in TBS, followed by incubation overnight with a primary antibody. 1:500 monoclonal rat anti-caspase-3, anti-b-actin (Santa Cruz Biotechnology Inc, CA, USA), and cleaved-caspase-3 (Cell Signaling Technology Inc, Danvers, Massachusetts, USA) were used. The incubation of the membrane was performed with HRP conjugated secondary antibody for two hours. Next, the membrane was developed with chemiluminescence reagents (Santa Cruz Biotechnology Inc, CA, USA). Afterwards, the membrane was exposed to Fuji Super RX film (Fujifilm Corporation, Tokyo, Japan). For the analysis of protein bands, "Image J Programme OD Analysis Software" was used (NIH, Bethesda, Maryland, USA). To normalize the signals, a respective $\beta$-actin signal was used (Çevik et al., 2012).

\section{Statistical analysis}

The Graphpad Prism 5.0 program was used for the statistical analysis (GraphPad Software, San Diego, CA, USA). All data were expressed as mean $\pm S D$. Biochemical data were analyzed with variance analysis (ANOVA) followed by Tukey multiple comparison tests. Statistical significance was accepted as $p<0.05$.

\section{RESULTS}

\section{Body weight and fasting blood glucose levels}

In the control group, body weight levels measured in the $8^{\text {th }}$ week were significantly increased as compared to basal values $(p<0.05)$ (Table 1). In the diabetes group, the 8th week body weight decreased significantly as compared to the baseline values $(p<0.001)$. In other groups, body weight levels did not demonstrate any significant difference.
The beginning (t1) blood glucose levels of all diabetic groups were significantly elevated with respect to that of control group ( $p<0.001$ ) (Table 2 ). Insulin, melatonin, and combined treatments reduced blood glucose levels at the end of the experiment (t2) with respect to t1 levels $(p<0.001)$.

\section{Intestinal tissue malondialdehyde (MDA) levels}

Intestinal tissue MDA levels were found to be significantly higher in the diabetes group as compared to the control group values $(p<0.001)$ (Figure 1). Insulin-alone administration did not improve the MDA levels with respect to the diabetes group and did not reduce the MDA levels to the control group levels $(p<0.05)$. Melatonin treatment given to diabetic rats abolished the increase in intestinal tissue MDA levels $(p<0.001)$. Combined treatment provided similar results $(p<0.001)$. In the combined treatment group, MDA levels were also demonstrated to be lower $(p<0.05)$ than insulin treatment alone.

\section{Intestinal tissue glutathione (GSH) levels}

Diabetes mellitus decreased intestinal tissue GSH levels significantly as compared to the control group levels $(p<0.001)$ (Figure 2). In the insulin treatment group, the GSH levels tended to increase with respect to the diabetes group; however, it was not statistically significant. Treatment of diabetic rats with either melatonin or insulin+melatonin elevated intestinal tissue GSH levels $(p<0.01-0.001)$.

\section{Intestinal tissue superoxide dismutase (SOD) levels}

Intestinal tissue SOD activity was decreased in diabetes and insulin-treated diabetes groups with respect to the control group ( $p<0.01$ and $p<0.05$ ) (Figure 3 ). Treatment with melatonin and insulin+melatonin increased the intestinal tissue SOD activity in the diabetic rats $(p<0.05)$

\section{Intestinal tissue myeloperoxidase (MPO) activity levels}

In the diabetes group, intestinal tissue MPO activity was higher than that of the control group $(p<0.001)$ (Figure 4). Myeloperoxidase activity was reduced in the insulin-treated group, but this decrease was not significant. Treatment of diabetic rats with either melatonin or melatonin+insulin abolished the MPO activity elevations $(p<0001)$.

\section{Intestinal tissue caspase-3 activity levels}

Intestinal tissue caspase-3 activity was elevated in the diabetes group compared with the control group $(p<0.01)$ (Figure 5). Insulin-alone administration did not significantly change caspase-3 activity levels. Melatonin and insulin+melatonin treatments decreased the intestinal tissue caspase-3 activity of diabetic animals $(p<0.05)$.

\section{Caspase-3 protein expression in intestinal tissue}

Caspase-3 bands increased significantly in the diabetic and insulin-treated animals with respect to the control group $(p<0.001)$. These bands decreased with the co-administration of melatonin and insulin $(p<0.001)$ (Figure 6). The combination of insulin and melatonin protected the cells against diabetesinduced damage through reducing cell death and increasing cell proliferation. 
Table 1. Body weights of the groups at the beginning and at the end of the experiment.

\begin{tabular}{|cccccc|} 
& \multicolumn{1}{c}{ C } & \multicolumn{1}{c}{ D } & \multicolumn{1}{c}{ D+I } & D+M & D+I+M \\
\hline $\mathbf{t}_{1}(\mathrm{~g})$ & $265 \pm 17$ & $257 \pm 15$ & $279 \pm 28.1$ & $266 \pm 15$ & $286 \pm 13$ \\
$\mathbf{t}_{2}(\mathrm{~g})$ & $312 \pm 9^{+}$ & $205 \pm 13^{+++}$ & $285 \pm 19$ & $288 \pm 21$ & $311 \pm 24$ \\
\hline
\end{tabular}

C: Control, D: Diabetes, I: Insulin, M: Melatonin. $t_{1}$ : at the beginning (after diabetes induction), $t_{2}$ : at $8^{\text {th }}$ week. Each group consists of 10 animals. Data are expressed as mean \pm SD. DATA are analyzed with variance analysis (ANOVA) followed by Tukey multiple comparison tests. ${ }^{+} p<0.05$, ${ }^{+++} \mathrm{p}<0.001$ : versus $t_{1}$.

Table 2. Fasting blood glucose levels of the groups at the beginning and at the end of the experiment.

\begin{tabular}{|c|c|c|c|c|c|}
\hline & C & D & $D+I$ & $D+M$ & $D+I+M$ \\
\hline$t_{1}(\mathrm{mg} / \mathrm{dL})$ & $98 \pm 12$ & $342 \pm 32^{\star \star \star}$ & $362 \pm 32^{\star \star \star}$ & $328 \pm 22^{\star \star \star}$ & $346 \pm 22^{\star * *}$ \\
\hline $\mathrm{t}_{2}(\mathrm{mg} / \mathrm{dL})$ & $93 \pm 10$ & $356 \pm 41$ & $116 \pm 18^{+++}$ & $181 \pm 16^{+++}$ & $102 \pm 10^{+++}$ \\
\hline
\end{tabular}

C: Control, D: Diabetes, I: Insulin, M: Melatonin. $\mathrm{t}_{1}$ : at the beginning (after diabetes induction), $\mathrm{t}_{2}$ : at $8^{\text {th }}$ week. Each group consists of 10 animals. Data are expressed as mean \pm SD. DATA are analyzed with variance analysis (ANOVA) followed by Tukey multiple comparison tests. ${ }^{* * *} p<0.001$ : versus control group at $\mathrm{t}_{1},{ }^{+++} \mathrm{p}<0.001$ : versus $\mathrm{t}_{1}$.

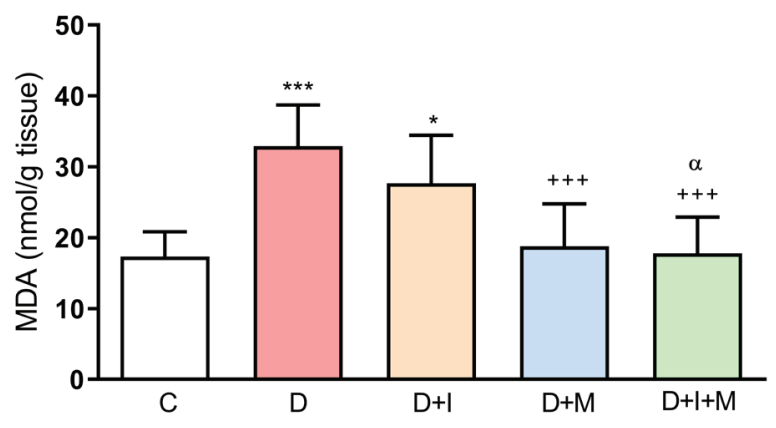

Figure 1. Malondialdehyde (MDA) levels in intestinal tissue of rats. ${ }^{*} p<0.05,{ }^{* * *} p<0.001$ : versus control group, $+++p<0.001$ : versus diabetes group, a $\mathrm{p}<0.05$ : versus insulin treated diabetes group. C: Control. D: Diabetes. I: Insulin. M: Melatonin. Each group consists of 10 animals. Data are expressed as mean \pm SD. DATA are analyzed with variance analysis (ANOVA) followed by Tukey multiple comparison tests.

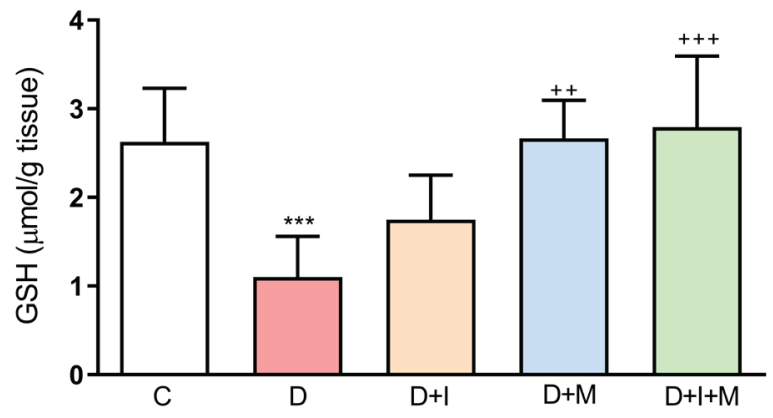

Figure 2. Glutathione $(\mathrm{GSH})$ levels in intestinal tissues of rats. ${ }^{* * *} \mathrm{p}<0.001$ : versus control group, $++p<0.01,+++p<0.001$ : versus diabetes group. C: Control. D: Diabetes. I: Insulin. M: Melatonin. Each group consists of 10 animals. Data are expressed as mean \pm SD. DATA are analyzed with variance analysis (ANOVA) followed by Tukey multiple comparison tests.

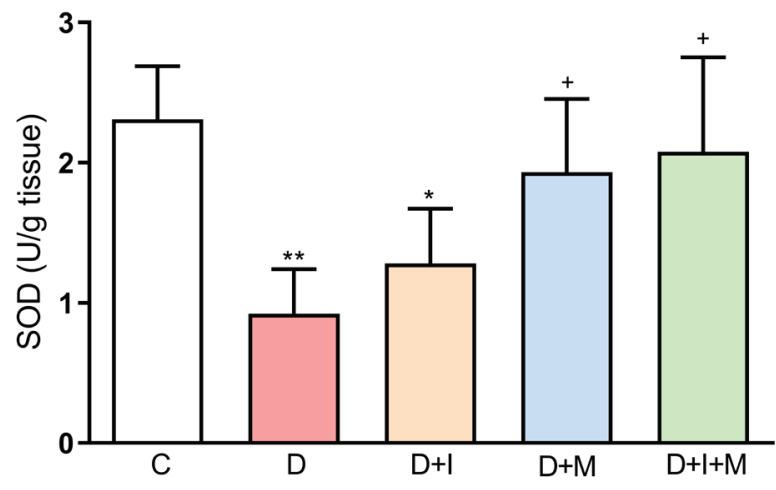

Figure 3. Superoxide dismutase (SOD) activities in intestinal tissues of rats. ${ }^{*} p<0.05,{ }^{* *} p<0.01$ : versus control group, $+p<0.05$ : versus diabetes group. C: Control. D: Diabetes. I: Insulin. M: Melatonin. Each group consists of 10 animals. Data are expressed as mean \pm SD. DATA are analyzed with variance analysis (ANOVA) followed by Tukey multiple comparison tests.

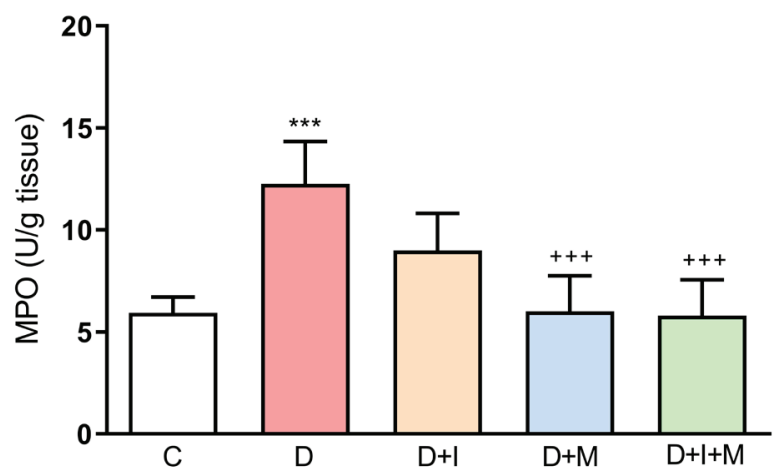

Figure 4. Myeloperoxidase (MPO) activities in intestinal tissues of rats. ${ }^{* * *} p<0.001$ : versus control group, $+++p<0.001$ : versus diabetes group. C: Control. D: Diabetes. I: Insulin. M: Melatonin. Each group consists of 10 animals. Data are expressed as mean \pm SD. DATA are analyzed with variance analysis (ANOVA) followed by Tukey multiple comparison tests. 


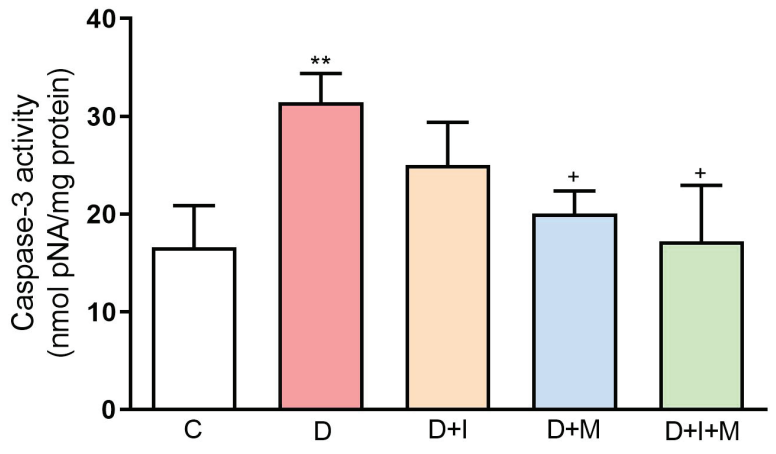

Figure 5. Caspase-3 activities in intestinal tissues of rats. ${ }^{* *} \mathrm{p}<0.01$ : versus control group, $+\mathrm{p}<0.05$ : versus diabetes group. C: Control. D: Diabetes. I: Insulin. M: Melatonin. Each group consists of 10 animals. Data are expressed as mean \pm SD. DATA are analyzed with variance analysis (ANOVA) followed by Tukey multiple comparison tests.
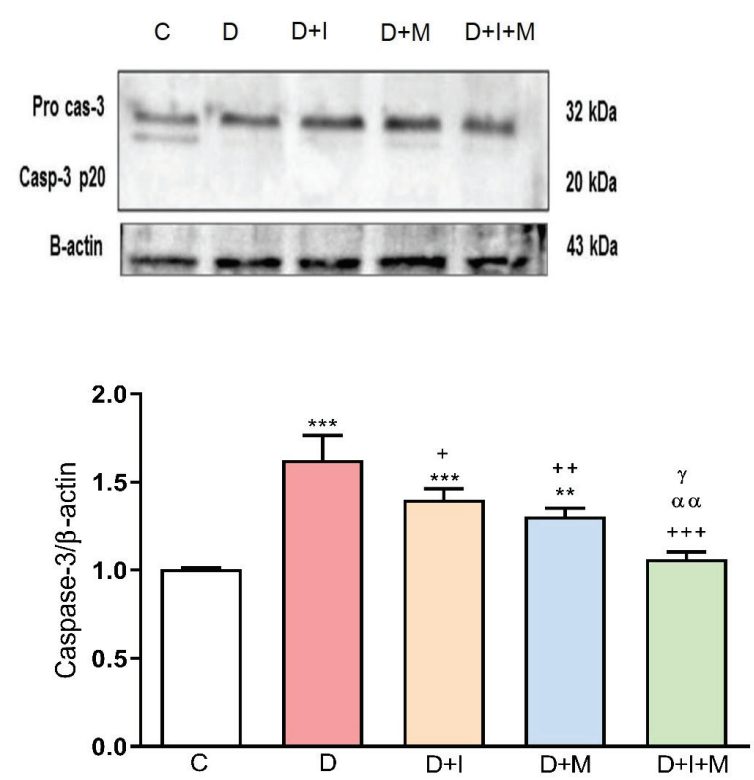

Figure 6. Caspase-3 protein expression in the intestinal tissues of rats determined by Western blotting. ${ }^{* *} \mathrm{p}<0.01$, ${ }^{* * *} p<0.001$ : versus control group, $+p<0.05,++p<0.01$, $+++p<0.001$ : versus diabetes group, $a a: p<0.01$ versus insulin treated diabetes group, $\gamma p<0.05$ : versus melatonin treated diabetes group. C: Control. D: Diabetes. I: Insulin. M: Melatonin. Each group consists of 10 animals. Data are expressed as mean \pm SD. DATA are analyzed with variance analysis (ANOVA) followed by Tukey multiple comparison tests.

\section{DISCUSSION}

Melatonin is a hormone secreted from the pineal gland, the main function of which is to regulate the circadian rhythm. Due to its lipophilic structure, melatonin is not stored in the pineal gland and diffuses into the blood. According to previous in vitro and in vivo experimental studies and clinical studies, melatonin has positive effects on glucose metabolism in DM (Owino, Buonfiglio, Tchio \& Tosini, 2019). In the culture study with pancreatic islet cells of type 2 diabetic patients, melatonin increased insulin release through repairing the $\beta$ cells (Costes, Boss, Thomas, \& Matveyenko, 2015). In the STZ-induced diabetes model, melatonin reduced fasting blood glucose levels (Alikhani et al., 2015). Additionally animals undergoing pinealectomy exhibited glucose tolerance impairment, insulin resistance, and DM, and these were repaired with melatonin treatment (Nogueira et al., 2011). In a clinical trial in type 2 diabetic patients, melatonin treatment reduced plasma glucose levels and provided glycemic control (Hussain et al., 2006). In accordance with previous reports, in our study, melatonin treatment significantly reduced the blood glucose levels in diabetic groups. Moreover, it potentialized the hypoglycemic effect of insulin in combined treatment group.

In DM, since glucose is spent for lipolysis and gluconeogenesis, the need of other cells for glucose is not adequately met and body weight decreases significantly (Alikhani et al., 2015; Husni et al., 2016). In our study, at the end of the experiment, it was demonstrated that the body weight levels were significantly lower in the diabetes group than the values at the beginning of the study. Insulin and melatonin treatments prevented the decrease in body weight.

Oxidative stress leads to serious complications in DM. In this regard, free radical formation are elevated, and antioxidant enzyme levels decrease in DM. Glucose oxidation produces radical anions, such as reactive ketoaldehydes and superoxide anions. Moreover, glucose reacts with proteins and generates advanced glycation end products that bind to their receptors and change the cellular functions (Vural et al., 2001; Hussain et al., 2006). The gastrointestinal tract is affected seriously by oxidative stress in DM. For instance, sensitivity of gastric mucosa to ulcers is elevated, gastric acid secretion is inhibited, or gastric mucosal atrophy arises (Alikhani et al., 2015).

Recent studies have drawn attention to melatonin as a free radical scavenger. In addition to directly neutralizing the free radicals, melatonin increases the effectiveness of antioxidants. It has been reported that melatonin possesses antioxidant activity in DM models through decreasing lipid peroxidation products and increasing antioxidant enzyme levels (Vural et al., 2001). Apart from the pineal gland, melatonin is also created in other organs, including the retina, Gl tract, and skin. The amount of $\mathrm{Gl}$ melatonin is 400 times higher than in the pineal gland (Yang et al., 2016). In addition to the introduction of circulating melatonin into the $\mathrm{Gl}$ tract, it is synthesized in the intestinal mucosa enterochromaphine cells of the Gl tract. All Gl tract tissues also have melatonin binding sites. Previously, Alikhani et al. showed that melatonin improved gastric mucus secretion and gastric motility in diabetic rats (Alikhani et al., 2015). Yang et al. demonstrated that melatonin restored elevated intestinal permeability in STZ-induced diabetic rats (Yang et al., 2016).

Malondialdehyde that is the final product of lipid peroxidation triggers atherosclerosis and atherotrombosis through oxidizing the LDL. In clinical researches, elevated plasma MDA levels were detected in diabetic patients (Mandal, Varghese, Gaviraju, Talwar, \& Malini, 2019). Hadjzadeh et al. demonstrated that 
melatonin treatment lowered gastric MDA levels and prevented gastric oxidative damage in STZ-induced diabetic rats (Hadjzadeh et al., 2018). Moreover, various studies exhibited positive effects of melatonin on elevated MDA levels in different tissues. For instance, Montilla et al. administered melatonin to the rats before triggering diabetes with STZ (Montilla, Tunez, de Agueda, Gascon, \& Soria, 1998). They detected that hyperglycemia and elevated MDA levels in plasma and renal tissue were prevented with melatonin treatment. In agreement with previous reports, in our study, melatonin-treated diabetic rats had significantly lower intestinal MDA levels compared to diabetic rats. In the combined treatment group, the MDA level also decreased significantly. Melatonin lifted the effectiveness of insulin in the combined treatment group.

With the thiol group, GSH eliminates the free radicals (Vural et al., 2001). According to experimental and clinical studies, DM affects GSH levels negatively due to its elevated oxidation or reduced synthesis. For instance, the clinical studies have shown that serum GSH concentration is reduced in DM patients (Mandal et al., 2019). Moreover, decreased intestinal GSH levels were determined with high glucose concentrations in the experimental studies (Bolkent, Bolkent, Yanardag, Mutlu, \& Yıldırım, 2006). The results of our study demonstrated that intestinal tissue GSH levels were significantly lower in the diabetes group than the control group. Both melatonin and combined treatments increased GSH levels compared to the diabetes group. Similarly, Vural et al. determined in their study conducted on STZ-induced diabetic rats that melatonin treatment brought decreased erythrocytes GSH levels to control group levels (Vural et al., 2001).

Superoxide dismutase, which is known as the antioxidant enzyme, catalyzes superoxide anion dismutation into oxygen and hydrogen peroxide. Loven et al. demonstrated in the experimental study that STZ-induced diabetic rats had reduced SOD levels in intestinal tissues (Loven et al., 1982). In various clinical studies, SOD levels were found to be low in DM patients due to SOD glucosylation and increased free radicals (Djordjević, Djurić, Djordjević, Apostolski, \& Živković, 2011). The results of our study demonstrated that diabetes reduced the SOD activity with respect to the control group. Superoxide dismutase activity was also lower in the insulin-treated group compared to the control group. However, SOD activity increased in the melatonin and combined treatment groups. Supportively, Hadjzadeh et al. demonstrated that melatonin treatment in STZ-induced diabetic rats improved gastric SOD activities (Hadjzadeh et al., 2018).

Myeloperoxidase is released from activated leukocytes in the inflammatory area and generates ROS (Garcia et al., 2015; Santhi, Shaik, \& Mahendran, 2017). Studies in diabetic patients have found that serum MPO levels increase with elevetad blood glucose levels (Song et al., 2015). High glucose levels increase hydrogen peroxide formation, and MPO uses hydrogen peroxide as a biological substrate to create highly reactive hypochlorid acid (Garcia et al., 2015). Furthermore, MPO triggers the formation of foam cells and plaque rupture through LDL modification. Supportively, it was demonstrated that coronary artery disease elevated plasma MPO concentration in diabetic patients (Song et al., 2015). Hardin et al. demonstrated elevated intestinal MPO activity in rats with Type 1 DM (Hardin, Donegan, Woodman, Trevenen, \& Gall, 2002). In different experimental studies, melatonin treatment has been shown to reduce MPO activity that was elevated in various tissues due to DM. For instance, Onk et al. found that melatonin treatment significantly reduced MPO levels in lung tissue that were elevated due to the STZ-induced diabetes in rats (Onk et al., 2018). Gurel-Gokmen et al. reported that melatonin treatment in STZinduced diabetic rats potentiates the effect of insulin to reduce MPO levels in brain tissue (Gurel-Gokmen et al., 2018). In our study, the diabetes group had significantly elevated MPO activity when compared to the control group. With melatonin and melatonin + insulin treatments, MPO activity decreased significantly.

Caspase-3, which is the mediator of cell apoptosis, is an enzyme member of the cysteine protease family (Ghosh et al., 2009). Various experimental studies suggested that high caspase- 3 activity contributes to both diabetes development and its complications. Liadis et al. found that diabetes did not occur in caspase-3 knockout mice with STZ administration because apoptosis did not develop in $\beta$ cells (Liadis et al., 2005). In different diabetes studies, increased caspase-3 activity was demonstrated to damage the hippocampus, kidneys, and retina through triggering apoptosis (Ghosh et al., 2009; Anarkooli, Sankian, Ahmadpor, Varasteh, \& Haghir, 2008; Kowluru \& Koppolu, 2002). In limited experimental studies, it has been shown that apoptosis due to high caspase-3 activity caused by hyperglycemia is alleviated with melatonin treatment (Onk et al., 2018). In our study, in diabetes group, intestinal caspase-3 activity was elevated significantly compared to control group. Melatonin treatment reduced caspase-3 activity compared to the diabetes group. This effect was not achieved with insulin therapy.

With the western blot method, it is possible to determine whether certain proteins specific to apoptosis are expressed or broken, such as caspase-3. In our study, when the expression of caspase-3 proteins were examined with western blot, it was demonstrated that diabetes and insulin treatment groups had increased caspasase-3 bands with respect to the control group. The reduction in these bands was observed with the co-administration of melatonin and insulin. It can be said that co-administration of insulin and melatonin decreases cell death and increases cell proliferation.

\section{CONCLUSION}

Our results demonstrate that melatonin treatment prevents the intestinal tissue damage in DM. Additionally antioxidant effects of melatonin on intestinal tissues are more obvious than insulin-alone therapy. As a consequence, melatonin could be used as a potential therapeutic supplement in diabetes-induced intestinal damage.

Acknowledgement: This study was funded by Marmara University Scientific Research Projects Committee with the grant number SAGC-YLP-091215-0532. 
Peer-review: Externally peer-reviewed.

Ethics Committee Approval: All experimental protocols were performed according to the Marmara University Animal Care and Use Committee (Protocol number: 33.2015.mar).

Author Contributions: Conception/Design of Study- Z.A.Ç.Y., G.B.A., Ö.Ç., G.Ş.; Data Acquisition- Z.A.Ç.Y, G.Ş.; Data Analysis/InterpretationZ.A.Ç.Y., G.Ş.; Drafting Manuscript- Z.A.Ç.Y., G.Ş.; Critical Revision of Manuscript- Z.A.Ç.Y., G.B.A, Ö.Ç, G.Ş; Final Approval and AccountabilityZ.A.Ç.Y., G.B.A, Ö.Ç, G.Ş; Technical or Material Support- Z.A.Ç.Y., G.B.A., Ö.Ç, G.Ş; Supervision- G.Ş.

Conflict of Interest: The authors have no conflict of interest to declare.

Financial Disclosure: This study was supported by Marmara University Scientific Research Porejects Committee (Grant number: SAG-CYLP-091215-0532).

\section{REFERENCES}

- $\quad$ Alikhani, V., Keshavarzi, Z., Hadjzadeh, M. A. R., \& Karimi, S. (2015). The effect of melatonin on gastric parameters following diabetes induction in male rats. Acta Endocrinologica (Buc), 11(2), 155-161. https://doi.org/10.4183/aeb.2015.155

- Anarkooli, I. J., Sankian, M., Ahmadpour, S., Varasteh, A. R., \& Haghir, H. (2008). Evaluation of Bcl-2 family gene expression and caspase-3 activity in hippocampus STZ- induced diabetic rats. Experimental Diabetes Research, 2008, 638467. https://doi. org/10.1155/2008/638467

- Bansal, A. K., \& Bilaspuri, G. S. (2011). Impact of oxidative stress and antioxidants on semen functions. Veterinary Medicine International, 2011, 686137. https://doi.org/10.4061/2011/686137

- Beuge, J. A., \& Aust, S. D. (1978). Microsomal lipid peroxidation. Methods in Enzymology, 52, 302-311. https://doi.org/10.1016/ s0076-6879(78)52032-6

- Beutler, E., Duron, O., \& Kelly, B. M. (1963). Improved method for the determination of blood glutathione. Journal of Laboratory and Clinical Medicine, 61, 882-888.

- Bhor, V. M., Raghuram, N., \& Sivakami, S. (2004). Oxidative damage and altered antioxidant enzyme activities in the small intestine of streptozotocin-induced diabetic rats. The International Journal of Biochemistry \& Cell Biology, 36, 89-97.

- Bolkent, S., Bolkent, S., Yanardag, R., Mutlu, O., \& Yildirim, S. (2006). Alterations in somatostatin cells and biochemical parameters following zinc supplementation in gastrointestinal tissue of streptozotocin-induced diabetic rats. Acta Histochem Cytochem, 39(1), 9-15. https://doi.org/10.1267/ahc.05054

- Bradford, M. M. (1976). A rapid and sensitive method for the quantitation of microgram quantities of protein utilizing the principle of protein-dye binding. Analytical Biochemistry, 72(2), 248-254. https://doi.org/10.1006/abio.1976.9999

- Costes, S., Boss, M., Thomas, A. P., \& Matveyenko, A. V. (2015). Activation of Melatonin Signaling Promotes $\beta$-Cell Survival and Function. Molecular Endocrinology, 29(5), 682-692. https://doi. org/10.1210/me.2014-1293

- Çevik, Ö., Oba, R., Macit, Ç., Çetinel, Ş., Çilingir-Kaya, Ö.T., Şener, E., \& Şener, G. (2012). Lycopene inhibits caspase-3 activity and reduces oxidativeorgan damage in a rat model of thermal injury. Burns, 38(6), 861-871. https://doi.org/10.1016/j.burns.2012.01.006

- Djordjevic, G. M., Djuric, S. S., Djordjevic, V. B., Apostolski, S., \& Zivkovic, M. (2011). The role of oxidative stress in pathogenesis of diabetic neuropathy: Erythrocyte superoxide dismutase, catalase and glutathione peroxidase level in relation to peripheral nerve conduction in diabetic neuropathy patients. Dr. Colleen Croniger (Ed.), Role of the Adipocyte in Development of Type 2 Diabetes (pp. 153-178). Rijeka, Croatia: InTech.. Retrieved from https://www.intechopen.com/ books/role-of-the-adipocyte-in-development-of-type-2-diabetes/ the-role-of-oxidative-stress-in-pathogenesis-of-diabetic-neuropathy-erythrocyte-superoxide-dismutase

- Esposito, E., \& Cuzzocrea, S. (2010). Antiinflammatory activity of melatonin in central nervous system. Current Neuropharmacology, 8(3), 228-242. https://doi.org/10.2174/157015910792246155

- $\quad$ Garcia, A. G., Rodrigues, M. R., Alonso, C. G., Rodrigues-Ochoa, D. Y., \& Aguilar, C. A. (2015). Myeloperoxidase is associated with insulin resistance and inflammation in overweight subjects with first-degree relatives with Type 2 diabetes mellitus. Diabetes \& Metabolism Journal, 39(1), 59-65. https://doi.org/10.4093/dmj.2015.39.1.59

- Ghosh, S., Khazaei, M., Moien-Afshari, F., Ang, L. S., Granville, D. J., Verchere, C. B. ... Laher, I. (2009). Moderate exercise attenuates caspase-3 activity, oxidative stress, and inhibits progression of diabetic renal disease in $\mathrm{db} / \mathrm{db}$ mice. American Journal of Physiology-Renal Physiology, 296(4), 700-708. https://doi.org/10.1152/ ajprenal.90548.2008

Gurel-Gokmen, B., Ipekci, H., Oktay, S., Alev, B., Ustundag, U. V., Ak, E., Tunali-Akbay, T. (2018). Melatonin improves hyperglycemia induced damages in rat brain. Diabetes Metabolism Research and Reviews, 34(8), e3060. https://doi.org/10.1002/dmrr.3060

Hadjzadeh, M. A. R., Alikhani, V., Hosseinian, S., Zarei, B., \& Keshavarzi, Z. (2018). The effect of melatonin against gastric oxidative stress and dyslipidemia in streptozotocin-ınduced diabetic rats. Acta Endocrinologica (Buchar), 14(4), 453-458. https://doi. org/10.4183/aeb.2018.453

- Hardin, J. A., Donegan, L., Woodman, R. C., Trevenen, C., \& Gall, D. G. (2002). Mucosal inflammation in a genetic model of spontaneous type I diabetes mellitus. Canadian Journal of Physiology and Pharmacology, 80(11), 1064-1070. https://doi.org/10.1139/y02-138

Hillegas, L. M., Griswold, D. E., Brickson, B., \& Winslow, C. A. (1990). Assessment of myeloperoxidase activity in whole rat kidney. Journal of Pharmacological Methods, 24(4), 285-295. https://doi. org/10.1016/0160-5402(90)90013-b

- Husni, A., Anggara, F. P., Isnansetyo, A., \& Nugroho, A. E. (2016). Blood glucose level and lipid profile of streptozotozin-induced diabetic rats treated with Sargassum polystum extract. International Journal of Pharmaceutical and Clinical Research, 8(5), 445450. https://doi.org/10.3923/jbs.2016.58.64

Hussain, S. A., Khadim, H. M., Khalaf, B. H., Ismail, S. H., Hussein, K. I., \& Sahib, A. S. (2006). Effects of melatonin and zinc on glycemic control in Type 2 diabetic patients poorly controlled with metformin. Saudi Medical Journal, 27(10),1483-1488.

Ighodaro, O. M. (2018). Molecular pathways associated with oxidative stress in diabetes mellitus. Biomedicine \& Pharmacotherapy, 108, 656-662.

- Kochar, N. I., \& Umathe, S. N. (2009). Beneficial effects of L-arginine against diabetes-induced oxidative stress in gastrointestinal tissues in rats. Pharmacological Reports, 61, 665-672. https://doi. org/10.1016/s1734-1140(09)70118-5

Kowluru, R., \& Koppolu, P. (2002). Diabetes-induced activation of caspase-3 in retina: effect of antioxidant therapy. Free Radical Research, 36(9), 993-999. https://doi.org/10.1080/ 1071576021000006572

- Liadis, N., Murakami, K., Eweida, M., Elford, A. R., Sheu, L., Gaisano, H. Y. ... Woo, M. (2005). Caspase-3-dependent $\beta$-cell apoptosis in the initiation of autoimmune diabetes Mellitus. Molecular and Cellular Biology, 25(9), 3620-3629. https://doi.org/10.1128/ MCB.25.9.3620-3629.2005

- $\quad$ Loven, D. P., Scheld, H. P., Oberley, L. W., Wilson, H. D., Bruch, L., Niehaus, C. L. (1982). Superoxide dismutase activity in the intestine of the streptozotocin-diabetic rat. Endocrinology, 111(3), 737-742. https://doi.org/10.1210/endo-111-3-737 
- Mandal, M., Varghese, A., Gaviraju, V. K., Talwar, S. N., Malini, S. S. (2019). Impact of hyperglycaemia on molecular markers of oxidative stress and antioxidants in type 2 diabetes mellitus. Clinical Diabetology, 8(4), 215-222. https://doi.org/10.5603/DK.2019.0015

- Montilla, P. L., Tunez, I. F., de Agueda, C. M., Gascon, F. L., Soria, J. V. (1998). Protective role of melatonin and retinol palmitate in oxidative stress and hyperlipidemic nephropathy induced by adriamycin in rats. Journal of Pineal Research, 25(2), 86-93. https://doi. org/10.1111/j.1600-079x.1998.tb00544.x

- Mylroie, A. A., Collins, H., Umbles, C., Kyle, J. (1986). Erythrocyte superoxide dismutase activity and other parameters of copper status in rats ingesting lead acetate. Toxicology and Applied Pharmacology, 82(3), 512-520. https://doi.org/10.1016/0041008x(86)90286-3

- Nogueira, T. C., Lellis-Santos, C., Jesus, D. S., Taneda, M., Rodrigues, S. C., Amaral, F. G. ... Anhe, G. F. (2011). Absence of melatonin induces night-time hepatic insulin resistance and increased gluconeogenesis due to stimulation of nocturnal unfolded protein response. Endocrinology, 152(4), 1253-1263. https://doi. org/10.1210/en.2010-1088

- Onk, D., Onk, O. A., Erol, H. S., Özkaraca, M., Çomaklı, S., Ayazoğlu, T. A., ... Ünver, S. (2018). Effect of melatonin on antioxidant capacity, Inflammation and apoptotic cell death in lung tissue of diabetic rats. Acta Cirurgica Brasileira, 33(4), 375-385. https://doi. org/10.1590/s0102-865020180040000009

- Owino, S., Buonfiglio, D. D. C., Tchio, C., Tosini, G. (2019). Melatonin signaling a key regulator of glucose homeostasis and energy metabolism. Frontiers in Endocrinology, 10, 488. https://doi. org/10.3389/fendo.2019.00488
Paskaloğlu, K., Şener, G., Ayanoğlu-Dülger, G. (2004). Melatonin treatment protects against diabetes-induced functional and biochemical changes in rat aorta and corpus cavernosum. European Journal of Pharmacology, 499(3), 345-354. https://doi. org/10.1016/j.ejphar.2004.08.002

- Santhi, T., Shaik, J. B., Mahendran, B. (2017). Myeloperoxidase levels predicts the vascular dysfunction in patients with Type 2 Diabetes Mellitus. Journal of Dental and Medical Sciences, 16(3), 30-34.

- $\quad$ Song, P., Xu, J., Song, Y., Jiang, S., Yuan, H., Zhang, X. (2015). Association of plasma myeloperoxidase level with risk of coronary artery disease in patients with Type 2 diabetes. Disease Markers, 2015, 761939. https://doi.org/10.1155/2015/761939

- Ullah, A., Khan, A., Khan, I. (2016). Diabetes mellitus and oxidative stress--A concise review. Saudi Pharmaceutical Journal, 24, 547-553. https://doi.org/10.1016/j.jsps.2015.03.013

- Vural, H., Sabuncu, T., Arslan, S.O., Aksoy, N. (2001). Melatonin inhibits lipid peroxidation and stimulates the antioxidant status of diabetic rats. Journal of Pineal Research, 31, 193-198. https://doi. org/10.1034/j.1600-079x.2001.310301.x

Wolosin, J. D., \& Edelman, S. V. (2000). Diabetes and the gastrointestinal tract. Clinical Diabetes, 18(4), 148.

- Yang, X., Zou, D., Tang, S., Fan, T., Su, H., Hu, R. ... Wang, Y. (2016). Ameliorative effect of melatonin against increased intestinal permeability in diabetic rats: possible involvement of MLCK-dependent MLC phosphorylation. Molecular Cellular Biochemistry, 416(12), 23-32. https://doi.org/10.1007/s11010-016-2691-4. 